\title{
A NEW TONOMETER BASED ON THE APPLICATION OF MICRO-MECHANICAL SENSORS
}

\author{
C. den Besten and P. Bergveld \\ MESA Research Institute, University of Twente \\ P.O.Box 217, 7500 AE Enschede \\ The Netherlands
}

\begin{abstract}
A new instrument for the measurement of intraocular pressure (IOP) is presented. It consists of a micro-machined silicon sensor, which measures the diameter of a flattened part of the eye globe and simultaneously determines the smallest distance between the centre of the sensor and the contour of this applanation. In the centre of this applanation sensor a micro-mechanical plunger is realised, which transfers the applied pressure to a separate force sensor.

Preliminary results show that this sensor may improve the accuracy of Mackay-Marg based electronic tonometers.
\end{abstract}

\section{INTRODUCTION}

About $3 \%$ of all people over the age of 40 suffer from the eye disease open-angle glaucoma [1]. This serious disease may lead to complete blindness without consecutive medical treatment.

An important indication for diagnosing open-angle glaucoma is an intraocular pressure (IOP) level of more than $20 \mathrm{mmHg}$, which is much higher than the average level of $10-15 \mathrm{mmHg}$. Therefore, ophthalmologists regularly measure the IOP of their patients with so-called tonometers.

Various electronic tonometers have been developed during the last decades, however, the accuracy of these tonometers is poor compared to conventional mechanical tonometers. In this paper a new electronic tonometer is presented, based on the application of two micro-mechanical sensors.

After the discussion of the measurement principle of Mackay and Marg, on which the new tonometer is based, the realisation and modelling of the sensor will be described. Finally preliminary results of experiments with this new tonometer will be presented.

\section{The MACKAY-MARg PRINCIPLE}

Mackay and Marg published in 1959 a paper in which they described methods to measure the IOP in an electronic way [2]. One of their proposals was a tonometer, which consisted of a footplate of $5 \mathrm{~mm}$ diameter with a small central pressure sensitive device as shown in figure 1 . The footplate is used to flatten the cornea of the eye, while the sensor output is monitored as a function of time. A dip in the pressure signal, which is electronically detected, indicates the actual IOP. This measurement principle, which is used in many electronic tonometers, is often called the MackayMarg principle.

It has been shown that the smallest distance between the contour of the applanation and the centre of the pressure sensor strongly influences the shape of the sensor output. When the contour of the applanation is located near the edge of the pressure sensitive area, there will be no dip in the sensor output. In that case the tonometer gives an erroneous readout [3].

Besides the location of the contour of the applanation with respect to the centre of the pressure sensor, the radius of the applanated area is also an important parameter in the measurement process. When the radius is too small capillary forces due to the tear film on the cornea and bending forces at the edge of the applanation caused by the stiffness of the cornea, will influence the measured

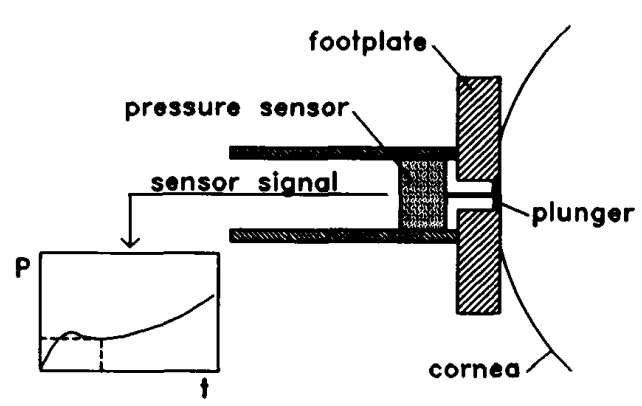

Figure 1: The Mackay-Marg principle. 
pressure. A too large radius causes a decrease in the internal volume of the eye globe resulting in an unwanted rise of the IOP.

However, radius nor distance between the contour of the flattened area and the centre of the pressure sensor are measured by Mackay-Marg-based tonometers. This might be a reason for their inaccuracy.

To solve this problem an applanation sensor is realised, which determines the radius of the applanation and also the smallest distance between the contour of the applanation and the centre of the sensor. A combination of this applanation sensor and a central pressure sensor will result in a modified Mackay-Marg tonometer, which may be more accurate due to the additional information about diameter and smallest distance between the pressure sensor and the contour of the flattened part of the eye.

\section{DESIGN OF THE APPLANATION SENSOR}

The design of the applanation sensor can be described as a three level structure separated by insulating layers. The first level consists of four arrays of meander-shaped diffused resistors realized around the centre of the silicon sensor as shown in figure 2 . The centre ends of the resistor arrays are commonly connected to the supply pad, while the other ends are separately connected to measurement pads. At equidistant positions metal meander contacts are made at the resistor nodes,

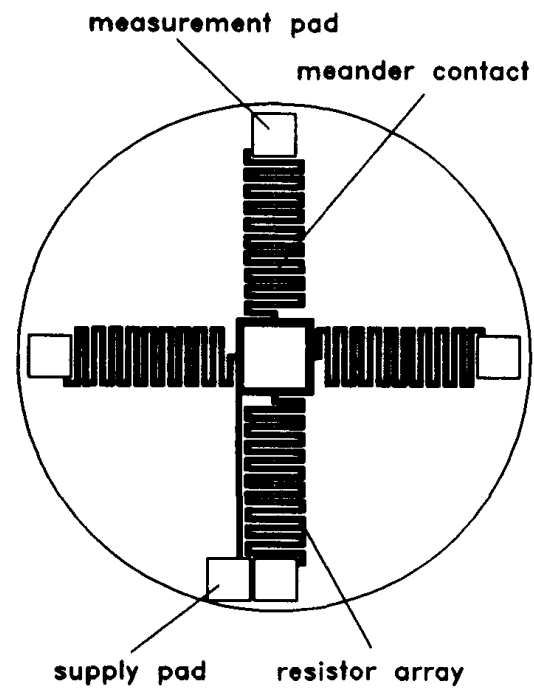

Figure 2: Top view of the first layer of the applanation sensor.

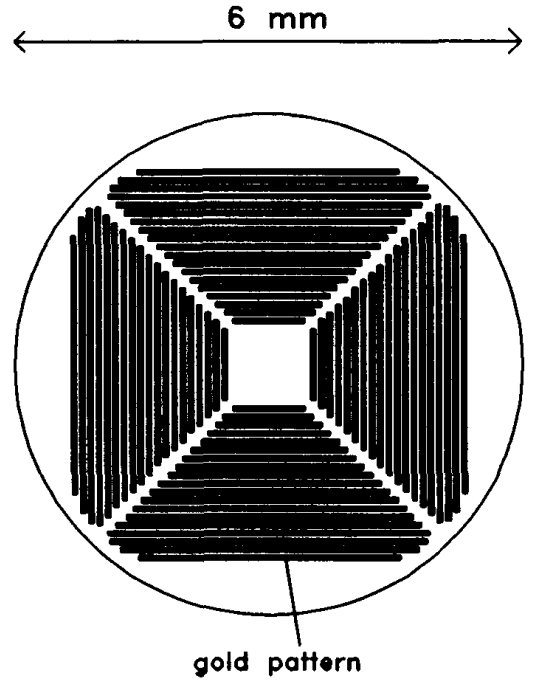

Figure 3: Top view of the gold pattern of the applanation sensor.

which connect the resistors through the insulating layer via contact holes to the second level. At that level for each array a gold pattern is realized, which consists of parallel separated lines as shown in figure 3. Each line is connected to one meander contact.

The third level consist of a Mylar foil, which is metallized at its underside and is separated from the second layer by an air-gap as shown in the cross section of the sensor in figure 4 . The gold film on the foil is electrically connected to the supply pad of the sensor.

The measurement principle is based on a change in resistance between the supply pad and the measurement pads of the four resistor arrays. When the sensor is used to flatten the eye, the foil is pressed against the gold pattern resulting in a short cut of parts of the resistor arrays. The change in resistance between the supply pad and the measurement pads is directly related to the diameter of the applanation and the smallest distance between the centre of the sensor and the contour of the applanation as will be shown in the next section.

If IOP-measurements based on the Mackay-Marg principle have to be performed, a pressure sensitive area must be realized in the centre of the applanation sensor. Although the integration of a pressure sensor would be preferable, we have decided to refrain from this task in order to focus 


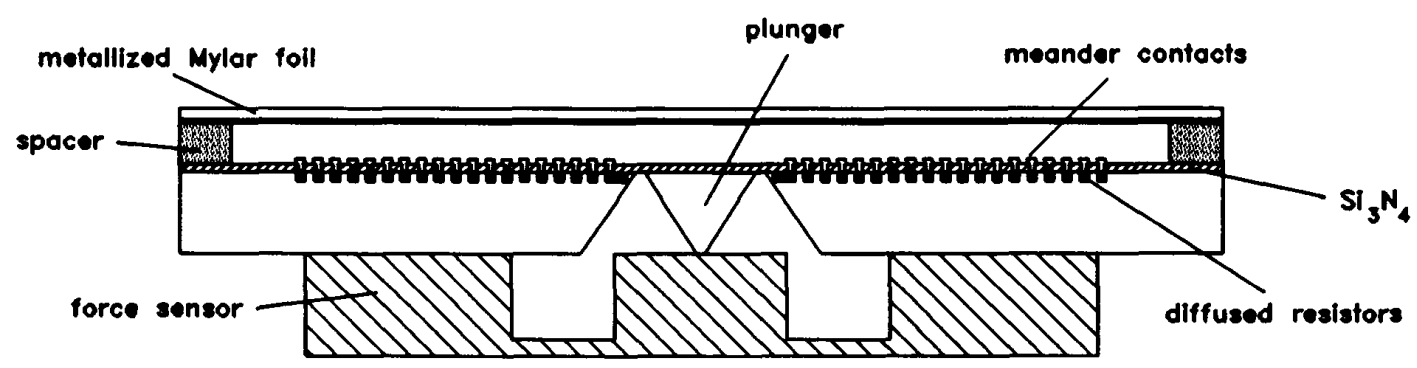

Figure 4: Cross section of the applanation sensor.

on the development of the applanation sensor. For the measurement of pressure commercially available sensors can be used if a plunger, which transfers the pressure from the plunger surface to a separated force sensor, is realised as shown in figure 4 .

\section{ELECTRICAL MODELLING}

The schematic electrical diagram of the applanation sensor is shown in figure 5. Each resistor array consists of $N$ contacts, spaced at a distance $d_{s}$, with a total resistance of $N R_{s}$. The separate resistors can be shunted by the conducting diaphragm, represented by the switches at the resistor nodes. The resistors $R_{c}$ and $R_{m}$ are serial resistances between the centre end of the arrays and the supply pad, and between the outer ends of the arrays and the measurement pads, respectively. The distance between the centre and the first switch of an array is noted as $d_{s o}$.

The distance $d_{n}$ between the centre of the sensor and the contour of the applanation can be calculated by equation (1), where the subscript $n$ refers to the number of the array and $R_{n}$ is the measured resistance between the supply pad and the measurement pad of that array.

$$
d_{n}=d_{s 0}+N d_{s}\left(1-\frac{R_{n}-R_{m}-R_{c}}{N R_{s}}\right)
$$

From the four distances $d_{n}$ the diameter $D$ and the smallest distance $d_{s m l}$ between the centre of the sensor and the contour of the applanation can be calculated by equations (2) and (3), respectively.

$$
D=2 d_{s 0}+N d_{s}\left(2-\frac{R_{1}+R_{3}-2 R_{c}-2 R_{m}}{N R_{s}}\right)
$$

$$
d_{s m l}=\frac{D}{2}-\frac{d_{s}}{2 R_{s}} \sqrt{\left(R_{3}-R_{1}\right)^{2}+\left(R_{4}-R_{2}\right)^{2}}
$$

It can be calculated that the error in $D$ and $d_{\text {sml }}$ due to the distance $d_{s}$ between two subsequent contacts will be less than $2 d_{s}$ and $3.5 d_{s}$, respectively.

In the actual realisation the pn-junctions between the resistors and the Si substrate and contact resistances between the $\mathrm{Si}$ of the resistor nodes and the Au-film of the Mylar foil have to be taken into account. However, it can be shown that the simplified model of figure 5 may be used as long as the contact resistances are much smaller than $\boldsymbol{R}_{s}$ and the diode reverse current $l_{s}$ is small compared to the total smallest current through the arrays.

\section{MECHANICAL MODELLING}

A mechanical model is given for the case that the

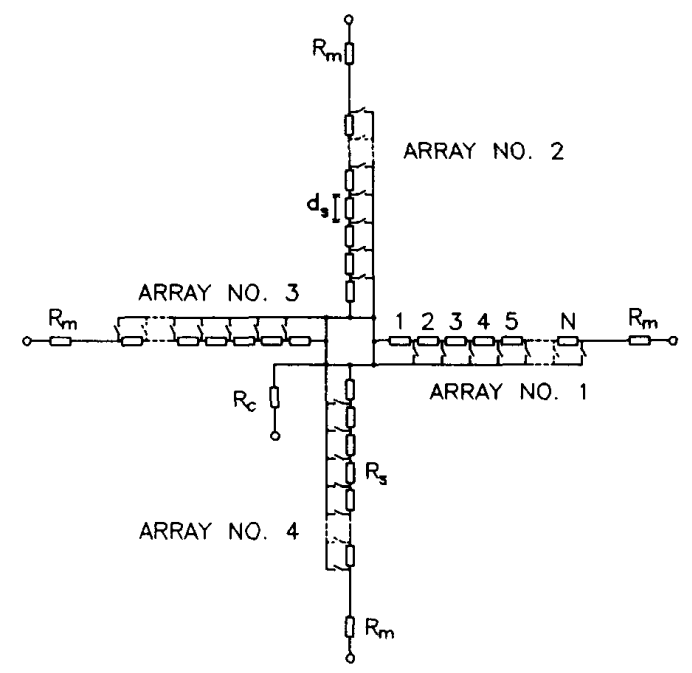

Figure 5: Electrical diagram of the applanation sensor. 
centre of the applanation coincides with the centre of the sensor. In all equations Poisson's ratio $v$ is assumed to be 0.3 .

The influence of the mechanical behaviour of the Mylar foil with thickness $t_{\text {foil }}$ and Young's modulus $E_{\text {foil }}$ of $3.8 \mathrm{e} 9 \mathrm{~N} / \mathrm{m}^{2}$ on the measured distance between the contour of the applanation and the centre of the sensor can be modelled by considering a circular diaphragm of radius $a$ with a central boss of radius $b$, which can be seen as the measured contour of the applanation. The force $F_{\text {foil }}$ required to realise a central deflection $y$, which corresponds to the thickness of the air-gap of the applanation sensor, can be calculated for $y \leq 0.5 t_{\text {foil }}$ and $t_{\text {foil }} \leq$ $0.25(a-b)$ by equation (4) [4].

$$
F_{\text {foil }}=\frac{4 \pi y E_{\text {foil }} t_{\text {foil }}{ }^{3}}{3\left(1-v^{2}\right)\left(a^{2}-b^{2}-\frac{4 a^{2} b^{2}}{a^{2}-b^{2}}\left(\ln \frac{a}{b}\right)^{2}\right)}
$$

The force $F_{\text {eye }}$, which can be exerted by the applanated cornea in order to press the Mylar foil against the gold pattern, can be calculated for 1.25 $<r<3.5 \mathrm{~mm}$ by equation (5) where $r$ is the radius of the applanation and $P{ }_{6}$ IOP [5]:

$$
F_{\text {eye }}=\pi r^{2}\left(P-\frac{0.9851}{r}+658.3\right)
$$

In figure 6 equations (4) and (5) are graphically represented. In curve $1 F_{\text {foil }}$, which is required to press a part of the foil with radius $b$ against the sensor surface, is shown as a function of the diameter of that area. In the calculations is assumed

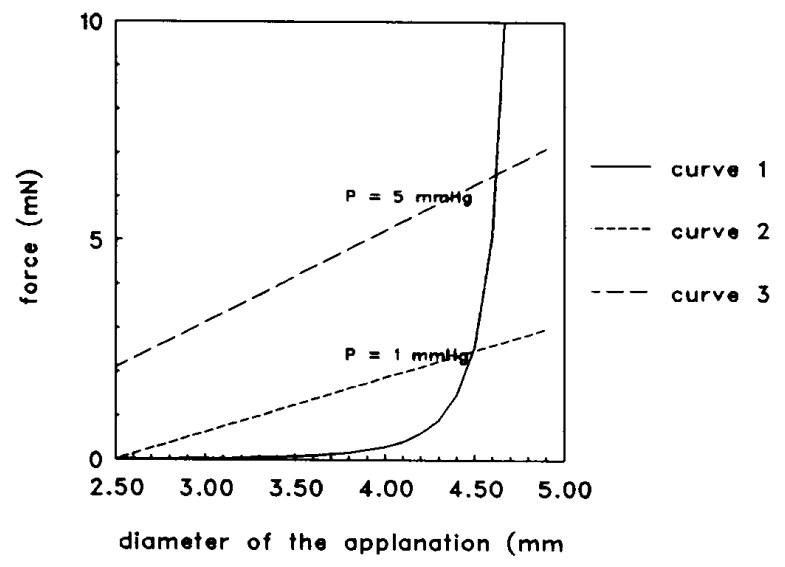

Figure 6: Graphical representation of equations (4) and (5). that a is $2.5 \mathrm{~mm}, y$ is $3 \mu \mathrm{m}$ and $t_{\text {foil }}$ is $6 \mu \mathrm{m}$.

In curve 2 and $3 F_{\text {eye }}$ is shown as a function of the diameter of the applanated area of the eye for an IOP of 1 and $5 \mathrm{mmHg}$, respectively. As long as $F_{\text {eye }} \geq F_{\text {foil }}$ the measured diameter will be correct, thus $r$ will be equal to $b$.

When a force sensor as shown in figure 4 is used the measured force depends on the mechanical behaviour of three diaphragms: the Mylar foil, the $\mathrm{Si}_{3} \mathrm{~N}_{4}$ diaphragm of the plunger and the diaphragm of the force sensor.

Assuming that the Mylar foil and the deflected plunger keep close contact, the force $F_{\text {appl }}$ required to realise a deflection $w$ of the plunger can be calculated by equation (6) [6]:

$$
F_{\text {appl }}=\frac{4 w p\left(E_{\text {foil }} t_{\text {foil }}{ }^{3}+E_{p l} t_{p l}^{3}\right)}{(p-q)^{2}}
$$

where $E_{p l}$ is the Young's modulus of the $\mathrm{Si}_{3} \mathrm{~N}_{4}$ diaphragm $\left(3 \mathrm{e} 11 \mathrm{Nm}^{-2}\right), t_{p l}$ is the thickness of the $\mathrm{Si}_{3} \mathrm{~N}_{4}$ diaphragm, $p$ is the outer radius of that diaphragm and $q$ is its inner radius.

The force $F_{f}$ required to realise the same deflection $w$ of the force sensor can be calculated by equation (7) [6]:

$$
F_{f}=\frac{4 \pi w E_{f} t_{f}^{3}}{3\left(1-v^{2}\right)\left(k^{2}-l^{2}-\frac{4 k^{2} l^{2}}{k^{2}-l^{2}}\left(\ln \frac{k}{l}\right)^{2}\right)}
$$

where $E_{f}$ is the Young's modulus of the force sensor diaphragm, $t_{f}$ is the thickness of that diaphragm, $k$ is the outer radius of the diaphragm and $l$ is the inner radius.

The relation between deflection $w$ of the complete configuration and the total applied force $F_{t o t}$ can be found by superposition of $F_{f}$ and $F_{a p p l}$.

\section{REALISATION}

The applanation sensor is made on a n-type silicon substrate. After depositing by CVD-techniques and patterning of doped oxide layers, the p-type resistors are formed as a result of a diffusion step at $1150^{\circ} \mathrm{C}$. Each resistor array consists of 72 resistors of $400 \mu \mathrm{m}$ long and $10 \mu \mathrm{m}$ wide. The boron dopant concentration of the resistors is about $3 \mathrm{e} 18$ $\mathrm{cm}^{-3}$ resulting in a resistance $R_{s}$ of $8 \mathrm{k} \Omega$. Highly boron doped areas are made at the nodes between 


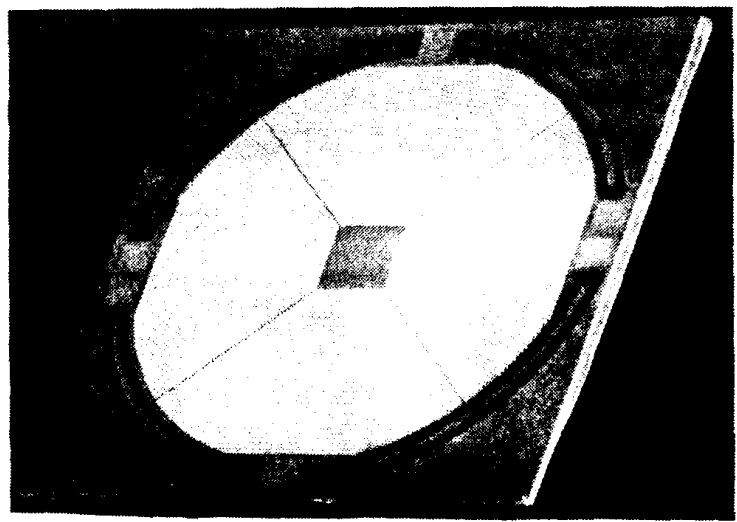

Figure 7: SEM photograph of the front side of the applanation sensor.

the resistors and at the pads in order to realise a low-ohmic contact between the metal contacts and the silicon. After the diffusion step the oxide layers are removed by wet chemical etching and a 750 $\mathrm{nm}$ thick LPCVD $\mathrm{Si}_{3} \mathrm{~N}_{4}$-layer is deposited, in which contact holes are etched at the highly doped areas of the resistors. Then a $1 \mu \mathrm{m}$ thick Al layer is evaporated, patterned and wet chemically etched, resulting in the supply and measurement pads and the meander contacts. The distance $d_{s}$ between two subsequent meander contacts is $30 \mu \mathrm{m}$, resulting in a maximum error of $60 \mu \mathrm{m}$ in the measured diameter of the applanation and $105 \mu \mathrm{m}$ in the calculated smallest distance between the contour of the applanation to the centre of the sensor.

After etching of the Al-layer a $25 \mathrm{~nm}$ Ti-layer and a $250 \mathrm{~nm}$ thick Au-layer are evaporated on top of the applanation sensor. The Ti-layer serves as an intermediate layer to improve the adhesion of the $\mathrm{Au}$ on the $\mathrm{Si}_{3} \mathrm{~N}_{4}$. The $\mathrm{Ti}$ and $\mathrm{Au}$-layers are patterned and etched by means of the IBE-technique.

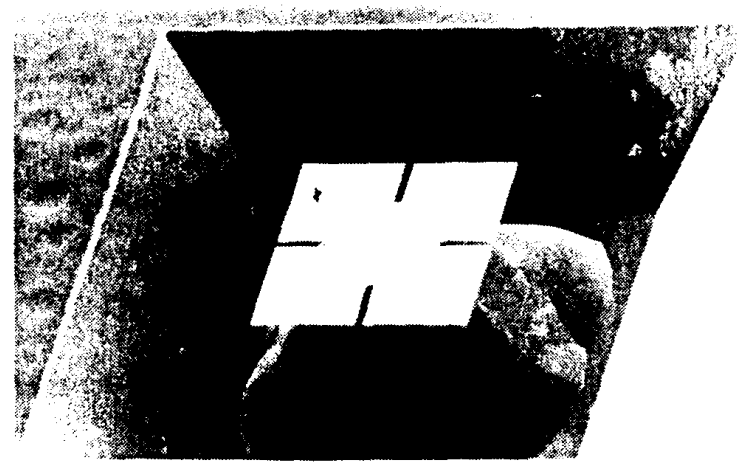

Figure 8: SEM photograph of the backside of the applanation sensor.
The resulting contact resistance between the Aulayer and the $\mathrm{Si}$ after an annealing step at $450^{\circ} \mathrm{C}$ for 20 minutes is less than $40 \Omega$.

Subsequently the spacer between the sensor surface and the Mylar foil is made by patterning and etching a $3 \mu \mathrm{m}$ thick polyimide layer. In figure 7 a SEM picture is shown of the completed front side of the applanation sensor before the Mylar foil is attached.

Finally the $\mathrm{Si}_{3} \mathrm{~N}_{4}$ on the backside of the wafer is patterned and etched to open etch holes for the etching of the plunger. The plunger is realised by etching the backside of the wafer in a $\mathrm{KOH}$ solution for approximately 7 hours at $70^{\circ} \mathrm{C}$. The $\mathrm{Si}_{3} \mathrm{~N}_{4}$ on the top of the wafer serves as the diaphragm for the plunger. The edge at the front side of the plunger is approximately $390 \mu \mathrm{m}$ long, the space between the contour of the plunger and the applanation sensor approximately $50 \mu \mathrm{m}$. Figure 8 shows a SEM-picture of the plunger seen from the backside of the sensor.

As the final step a $6 \mu \mathrm{m}$ thick Mylar foil covered at one side with a $50 \mathrm{~nm}$ layer of gold, which is externally connected to the supply pad, is attached to the sensor by means of glue.

\section{EXPERIMENTAL RESULTS}

To test the applanation sensor an artificial eye has been made consisting of a fluid-filled pressure chamber covered by a $25 \mu \mathrm{m}$ thick rubber foil. The pressure inside the chamber can be adjusted by a column of water.

In the first experiment the entire applanation sensor was mounted on a force sensor. The quotient

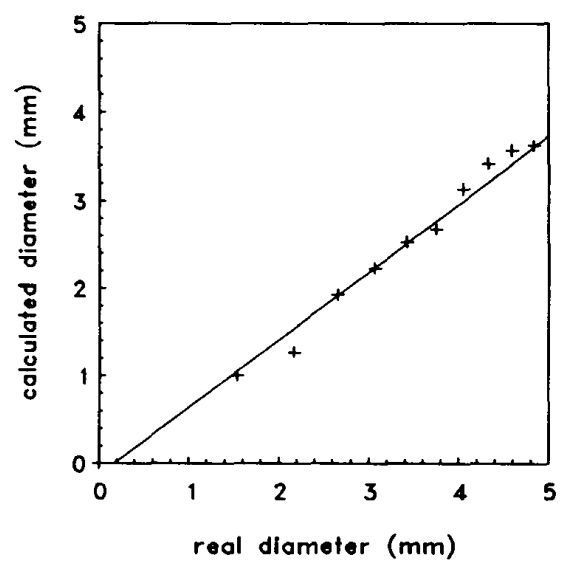

Figure 9: Results of the diameter measurement. 


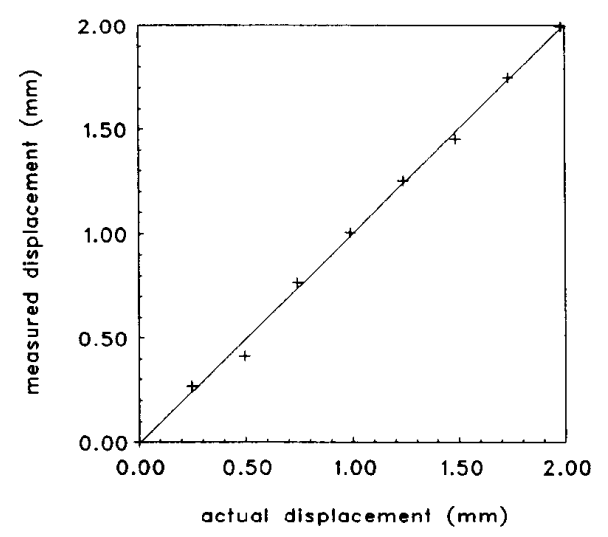

Figure 10: Results of the measurement of displacement.

of the force, which is used to flatten the eye and the internal pressure is equal to the area of the applanation, from which the real diameter of the applanation can be calculated. In figure 9 the diameter calculated by equation (2) is shown as a function of the real diameter. From the figure it can be concluded that there is a linear relation between the calculated and the real diameter. The real diameter is always larger than the calculated diameter. This may be caused by the relative thick air-gap $(100 \mu \mathrm{m})$ due to problems during glueing of the Mylar diaphragm.

To test the measurement of the distance between the centre of the sensor and the contour of an applanation a thin shaft of air was used to press the diaphragm at a located spot against the sensor surface. By moving the spot with a micro-manipulator

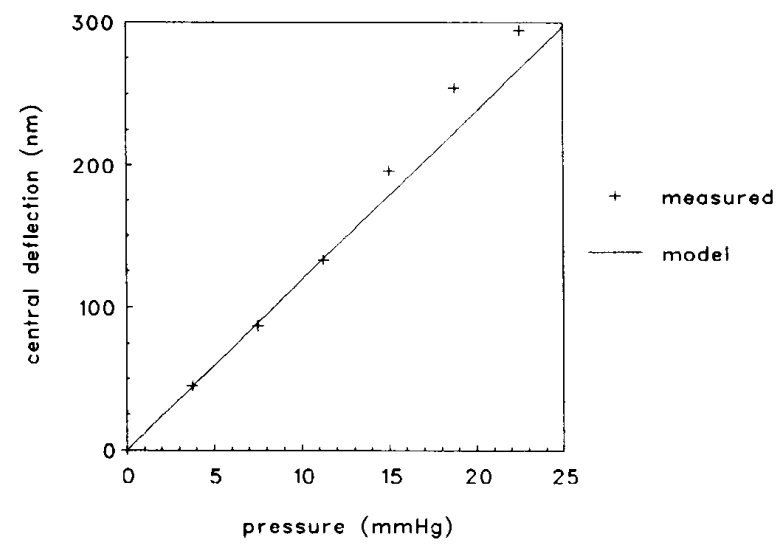

Figure 11: Deflection of the plunger as a function of the applied pressure. along the arrays the measured displacement could be compared to the actual displacement given by the micro-manipulator. A typical result for an array is shown in figure 10 .

It can be seen that there is an excellent linear relation between the measured and actual displacement. The small irregularities may be caused by dust particles between the foil and the sensor surface.

The deflection of the plunger is measured as a function of a uniform applied force. The results are shown in figure 11 . The theoretical relation calculated by equation (6) is indicated by the solid line. The small deviation between theory and measurements may be caused by a small difference in Young's modulus and/or diaphragm thickness between the assumed values in the model and the practical realisation.

\section{Conclusions}

A new tonometer based on two micro-mechanical sensors is presented. From the results presented in the previous section it may be concluded that the applanation sensor can be used to measure the diameter of an applanation and the distance between the contour of the applanation and the centre of the sensor. The mechanical behaviour of the plunger complies well with the theoretical description. However, further research is required to investigate the mechanical behaviour of the total tonometer configuration.

\section{REFERENCES}

[1] D. Robertson, "Tonometry screening on the medical service", Arch. Intern. Med., Vol. 137 pp. 443-445: 1977

[2] R.W. Mackay and E. Marg, 'Fast automatic electronic tonometers based on an exact theory", Acta Ophth., Vol. 37 pp. 459-507: 1959

[3] J. Stepanik, "Das Mackay-Marg-Tonometer. 3. Das Verhalten des Tonogramms bei zunehmendem Dezentrieren der Sonde gegenüber der Hornhautapplanation", Albr. von Graefes Arch. Klin. Exp. Ophth., Vol. 182 pp. 144-146: 1971

[4] R.J. Roark, "Formulas for stress and strain", New York: McGraw-Hill: 1954

[5] H. Goldmann and Th. Schmidt, "Weiterer Beitrag zur Applanationstonometrie", Ophthal., Vol. 144 pp. 441456: 1961

[6] P. Ohlckers, O. Eriksen and H. Jenssen, " A mechanical silicon sensor element with low deflection and high resonance frequency", proc. Transducers'87, pp. 332335: 1987 